\title{
Metal-Ligand Cooperation in $\mathrm{C}-\mathrm{H}$ and $\mathrm{H}_{2}$ Activation by an Electron- Rich PNP Ir(I) System. Ligand Dearomatization - Aromatization as
} Key Steps

\author{
Eyal Ben-Ari, Gregory Leitus, Linda J. W. Shimon and David Milstein* \\ Department of Organic Chemistry and Unit of Chemical Research \\ Support, Weizmann Institute of Science, Rehovot, 76100 Israel
}

General procedures. All experiments with metal complexes and phosphine ligands were carried out under an atmosphere of purified nitrogen in a Vacuum Atmospheres glove box equipped with a MO 40-2 inert gas purifier or using standard Schlenk techniques. All solvents were reagent grade or better. All non-deuterated solvents were refluxed over sodium/benzophenone ketyl and distilled under argon atmosphere. Deuterated solvents were used as received. All the solvents were degassed with argon and kept in the glove box over 4A molecular sieves. Commercially available reagents were used as received. ${ }^{1} \mathrm{H},{ }^{13} \mathrm{C},{ }^{31} \mathrm{P}$ and ${ }^{19} \mathrm{~F}$ NMR spectra were recorded at 400, 100, 162 and $376 \mathrm{MHz}$, respectively, using a Bruker AMX-400 NMR spectrometer and at 500, 125 and $202 \mathrm{MHz}$, respectively, for ${ }^{1} \mathrm{H},{ }^{13} \mathrm{C},{ }^{31} \mathrm{P}$, using a Bruker Avance-500 NMR spectrometer. All spectra were recorded at $23{ }^{\circ} \mathrm{C}$. ${ }^{1} \mathrm{H}$ NMR and ${ }^{13} \mathrm{C}\left\{{ }^{1} \mathrm{H}\right\}$ NMR chemical shifts are reported in ppm downfield from tetramethylsilane. 'H NMR chemical shifts were referenced to the residual hydrogen signal of the deuterated solvents (7.15 ppm, benzene; $3.58 \mathrm{ppm}$ and $1.73 \mathrm{ppm}$, tetrahydrofuran). In ${ }^{13} \mathrm{C}\left\{{ }^{1} \mathrm{H}\right\} \mathrm{NMR}$ measurements the signals of benzene-d6 (128.1 ppm) and THF (67.4 ppm, $25.3 \mathrm{ppm})$ were used as a reference. ${ }^{31} \mathrm{P}$ NMR chemical shifts are reported in ppm downfield from $\mathrm{H}_{3} \mathrm{PO}_{4}$ and referenced to an external $85 \%$ solution of phosphoric acid in $\mathrm{D}_{2} \mathrm{O}$. Abbreviations used in the description of NMR data are as follows: b, broad; s, singlet; $d$, doublet; $t$, triplet; q, quartet, m, multiplet, v, virtual. 


\section{Reaction of $[\operatorname{Ir}(\mathrm{PNP})(\mathrm{COE})]\left[\mathrm{PF}_{6}\right]$ (1) with ${ }^{\mathrm{t}} \mathrm{BuOK}$. Formation of $\operatorname{Ir}\left(\mathrm{PNP}^{*}\right) \mathrm{COE}$ (2)}

To a solution $(2 \mathrm{~mL})$ of $[\operatorname{Ir}(\mathrm{PNP})(\mathrm{COE})]\left[\mathrm{PF}_{6}\right] 1(15 \mathrm{mg}, 0.039 \mathrm{mmol})$ in THF $(2 \mathrm{~mL})$ was added ${ }^{\mathrm{t}} \mathrm{BuOK}$ (4.4 mg, $0.039 \mathrm{mmol}$ ). The color changed to purple immediately. The solvent was evaporated and the resulting purple solid was dissolved in pentane and filtered through a fine $(0.2 \mu \mathrm{m})$ Teflon filter. Solvent evaporation resulted in complex 2 as purple solid in nearly quantitative yield.

2: ${ }^{31} \mathrm{P}\left\{{ }^{1} \mathrm{H}\right\}$ NMR $\left(\mathrm{C}_{6} \mathrm{D}_{6}\right): 34.00$ (A/B quartet, $\left.{ }^{2} \mathrm{~J}_{\mathrm{PP}}=336.0 \mathrm{~Hz}\right) .{ }^{1} \mathrm{H}$ NMR $\left(500 \mathrm{MHz}, \mathrm{C}_{6} \mathrm{D}_{6}\right)$ $1.20\left(\mathrm{~m}, \quad 18 \mathrm{H}, \quad \mathrm{P}-\mathrm{C}\left(\mathrm{CH}_{3}\right)_{3}\right), 1.42\left(\mathrm{~m}, \quad 18 \mathrm{H}, \quad \mathrm{P}-\mathrm{C}\left(\mathrm{CH}_{3}\right)_{3}\right), 1.65(\mathrm{~m}, \quad 10 \mathrm{H}, \quad \mathrm{COE}$, $\left.\mathrm{CH}_{2} \mathrm{CH}_{2} \mathrm{CH}=\mathrm{CH}\right), 2.47\left(\mathrm{~m}, 2 \mathrm{H}, \mathrm{CH}_{2} \mathrm{CH}=\mathrm{CH}\right) 2.8\left(\mathrm{~d}, 2 \mathrm{H},{ }^{2} \mathrm{~J}_{\mathrm{PH}}=10 \mathrm{~Hz}, \mathrm{P}-\mathrm{CH}_{2} \mathrm{Py}\right), 3.68(\mathrm{~m}$, $2 \mathrm{H}, \mathrm{COE}, \mathrm{CH}=\mathrm{CH}), 3.75\left(\mathrm{vt}, 1 \mathrm{H},{ }^{2} \mathrm{~J}_{\mathrm{PH}}=6 \mathrm{~Hz}, \mathrm{P}-\mathrm{CHPy}\right), 5.4\left(\mathrm{br} \mathrm{d},{ }^{3} \mathrm{~J}_{\mathrm{HH}}=6 \mathrm{~Hz}, \mathrm{Py}-H\right), 6.35$ $(\mathrm{m}, 2 \mathrm{H}, \mathrm{Py}-H) 6.43(\mathrm{~m}, 1 \mathrm{H}, \mathrm{Py}-H){ }^{13} \mathrm{C}\left\{{ }^{1} \mathrm{H}\right\}$ NMR (500 MHz, $\left.\mathrm{C}_{6} \mathrm{D}_{6}\right): 27.3$ (s, COE), 30 (d, $\left.{ }^{2} \mathrm{~J}_{\mathrm{PC}}=6.25 \mathrm{~Hz}, \mathrm{P}-\mathrm{C}\left(\mathrm{CH}_{3}\right)_{3}\right), 30.4-31\left(\mathrm{~m}, \mathrm{P}-\mathrm{C}\left(\mathrm{CH}_{3}\right)_{3}\right), 33\left(\mathrm{~s}, \mathrm{CH}_{2} \mathrm{CH}=\mathrm{CH}\right), 35\left(\mathrm{vt},{ }^{2} \mathrm{~J}_{\mathrm{PC}}=9.3 \mathrm{~Hz}\right.$, P-C $\left.\left(\mathrm{CH}_{3}\right)_{3}\right), 36.7\left(\mathrm{~m}, \mathrm{CH}_{2} \mathrm{P}\right), 37.4\left(\mathrm{~m}, \mathrm{P}-\mathrm{C}\left(\mathrm{CH}_{3}\right)_{3}\right), 40.2\left(\mathrm{~d},{ }^{2} \mathrm{~J}_{\mathrm{PC}}=6.25 \mathrm{~Hz}, \mathrm{CH}_{2} \mathrm{P}\right) 71.5(\mathrm{dd}$, $\left.{ }^{2} \mathrm{~J}_{\mathrm{PC}}=9.3 \mathrm{~Hz}, \mathrm{P}-\mathrm{CHPy}\right), 96(\mathrm{~s}, \mathrm{COE}, \mathrm{HC}=\mathrm{CH}), 115\left(\mathrm{~d},{ }^{2} \mathrm{~J}_{\mathrm{PC}}=12 \mathrm{~Hz}, \mathrm{Py}-\mathrm{CH}\right), 128.3(\mathrm{~s}, \mathrm{Py}-\mathrm{CH})$, 128.55 (s, Py-CH), 131.57 (s, Py-CH), 159.5 (s, Py-CC ), 172.5 (m, Py-CC) ; ${ }^{15} \mathrm{~N}-\mathrm{H}$ correlation NMR in $\mathrm{C}_{6} \mathrm{D}_{6}$ : the protons at $2.8 \mathrm{ppm}$ and $3.75 \mathrm{ppm}$ were correlated to a ${ }^{15} \mathrm{~N}$ signal at $194 \mathrm{ppm}$. The structure was confirmed by DEPT, C-H correlation experiment and 2D COSY NMR.

\section{Reaction of $\operatorname{Ir}\left(\mathrm{PNP}^{*}\right) \mathrm{COE}(2)$ with benzene. Formation of $\operatorname{Ir}(\mathrm{PNP})\left(\mathrm{C}_{6} \mathrm{H}_{5}\right)(4)$.}

A benzene solution of $\operatorname{Ir}\left(\mathrm{PNP}^{*}\right)(\mathrm{COE}) 2$ was heated to $60{ }^{\circ} \mathrm{C}$ for $2 \mathrm{~h}$ after which the color changed from purple-red to dark grey-violet. Evaporation of the solvent resulted in complex 4 as greyish solid in quantitative yield. Alternatively, reacting complex $\left[\operatorname{Ir}(\mathrm{PNP})(\mathrm{H})\left(\mathrm{C}_{6} \mathrm{H}_{5}\right)\right] \mathrm{PF}_{6} 5(15 \mathrm{mg}, 0.0185 \mathrm{mmol})$ with ${ }^{\mathrm{t}} \mathrm{BuOK}(2 \mathrm{mg}, 0.0185 \mathrm{mmol})$ at $25{ }^{\circ} \mathrm{C}$ resulted in complex 4 in nearly quantitative yield. 4: ${ }^{31} \mathrm{P}\left\{{ }^{1} \mathrm{H}\right\}$ NMR $\left(\mathrm{C}_{6} \mathrm{D}_{6}\right): 55.00(\mathrm{~s}) .{ }^{1} \mathrm{H}$ NMR (500 MHz, $\left.\mathrm{C}_{6} \mathrm{D}_{6}\right) 1.25\left(\mathrm{vt}, 36 \mathrm{H},{ }^{3} \mathrm{~J}_{\mathrm{PH}}=6.5 \mathrm{~Hz}, \mathrm{P}-\mathrm{C}\left(\mathrm{CH}_{3}\right)_{3}\right), 2.37$ (vt, 4H, ${ }^{2} \mathrm{~J}_{\mathrm{PH}}=3 \mathrm{~Hz}, \mathrm{P}-$ $\left.\mathrm{CH}_{2} \mathrm{Py}\right), 6.36\left(\mathrm{~d}, 2 \mathrm{H},{ }^{3} \mathrm{~J}_{\mathrm{HH}}=7.5 \mathrm{~Hz}, \mathrm{Py}-\mathrm{H}\right), 6.90\left(\mathrm{t}, 1 \mathrm{H},{ }^{3} \mathrm{~J}_{\mathrm{HH}}=7.0 \mathrm{~Hz}\right.$, Aryl-H), $7.27(\mathrm{t}, 1 \mathrm{H}$, ${ }^{3} \mathrm{~J}_{\mathrm{HH}}=7.5 \mathrm{~Hz}$, Aryl-H), $7.65\left(\mathrm{t}, 1 \mathrm{H},{ }^{3} \mathrm{~J}_{\mathrm{HH}}=7.5 \mathrm{~Hz}\right.$, Py- $\left.\mathrm{H}\right), 7.99\left(\mathrm{~d}, 2 \mathrm{H},{ }^{3} \mathrm{~J}_{\mathrm{HH}}=7.0 \mathrm{~Hz}\right.$, Aryl$H) .{ }^{13} \mathrm{C}\left\{{ }^{1} \mathrm{H}\right\}$ NMR $\left(500 \mathrm{MHz}, \mathrm{C}_{6} \mathrm{D}_{6}\right): 29.45\left(\mathrm{~m}, \mathrm{P}-\mathrm{C}\left(\mathrm{CH}_{3}\right)_{3}\right), 36.20$ (vt, ${ }^{2} \mathrm{~J}_{\mathrm{PC}}=8.75 \mathrm{~Hz}, \mathrm{P}-$ $\left.\mathrm{C}\left(\mathrm{CH}_{3}\right)_{3}\right), 39.60\left(\mathrm{vt},{ }^{2} \mathrm{~J}_{\mathrm{PC}}=8.75 \mathrm{~Hz}, \mathrm{CH}_{2} \mathrm{P}\right), 118.32(\mathrm{~s}, \mathrm{Py}-\mathrm{CH}), 120.28\left(\mathrm{vt},{ }^{2} \mathrm{~J}_{\mathrm{PC}}=5.0 \mathrm{~Hz}\right.$, Aryl$\mathrm{CH}), 125.9$ (s, Py-CH), 127.18 (s, Aryl-CH), 144.9 (s, Aryl-CH ), 153.5 (vt, ${ }^{2} \mathrm{~J}_{\mathrm{PC}}=8.75 \mathrm{~Hz}$, $\left.\mathrm{C}_{\mathrm{ipso}}\right) 161.90\left(\mathrm{vt},{ }^{2} \mathrm{~J}_{\mathrm{PC}}=5.0 \mathrm{~Hz}, \mathrm{Py}-\mathrm{CH}\right) ;{ }^{15} \mathrm{~N}-\mathrm{H}$ correlation NMR in $\mathrm{C}_{6} \mathrm{D}_{6}$ : the protons at 2.37 ppm were correlated to an ${ }^{15} \mathrm{~N}$ signal at $277 \mathrm{ppm}$. The structure was confirmed by DEPT, C$\mathrm{H}$ correlation experiment and 2D COSY NMR. Elemental analysis of 4: Calc. for $\mathrm{C}_{29} \mathrm{H}_{48} \mathrm{P}_{2} \mathrm{NIr}$ : C, 52.39; H, 7.28; Found: C, 52.26; H, 7.28. 


\section{Reaction of $\left[\operatorname{Ir}(\mathrm{PNP})(\mathrm{H})\left(\mathrm{C}_{6} \mathrm{H}_{5}\right)\right]\left[{ }^{+} \mathrm{PF}_{6}^{-}\right]$(5) with ${ }^{\mathrm{t}} \mathrm{BuOK}$ at $-78^{\circ} \mathrm{C}$. Formation of $\operatorname{Ir}\left(\mathrm{PNP}^{*}\right)\left(\mathrm{C}_{6} \mathrm{H}_{6}\right)(\mathrm{H})(3)$ and $\operatorname{Ir}(\mathrm{PNP})\left(\mathrm{C}_{6} \mathrm{H}_{5}\right)(4)$.}

To a THF solution of $\left[\operatorname{Ir}(\mathrm{PNP})(\mathrm{H})\left(\mathrm{C}_{6} \mathrm{H}_{5}\right)\right]\left[\mathrm{PF}_{6}\right] 5(15 \mathrm{mg}, 0.0185 \mathrm{mmol})$ at $-78{ }^{\circ} \mathrm{C}$ was added ${ }^{\mathrm{t}} \mathrm{BuOK}$ (potassium tert-butoxide) $(2 \mathrm{mg}, 0.0185 \mathrm{mmol})$ the color immediately changed to dark brown. ${ }^{31} \mathrm{P}$ NMR revealed an $\mathrm{A} / \mathrm{B}$ quartet implying the lack of a symmetry plane resulting from the deprotonation of the benzylic protons. ${ }^{1} \mathrm{H}$ NMR revealed a hydride at -47 ppm typical for an hydride trans to a vacant coordination site. Signals at $5.67 \mathrm{ppm}$ and 6.45$6.85 \mathrm{ppm}$ are indicative to the dearomatization of the PNP ligand. Warming the solution to room temperature resulted after few seconds in formation of clean complex 4.

3: ${ }^{31} \mathrm{P}\left\{{ }^{1} \mathrm{H}\right\}$ NMR (THF d,$\left.-78{ }^{\circ} \mathrm{C}\right): 53.0\left(\mathrm{~d},{ }^{2} \mathrm{~J}_{\mathrm{PP}}=336 \mathrm{~Hz}, P-\mathrm{Ir}-\mathrm{P}\right), 43.0\left(\mathrm{~d},{ }^{2} \mathrm{~J}_{\mathrm{PP}}=336 \mathrm{~Hz}, \mathrm{P}-\right.$ Ir-P). ${ }^{1} \mathrm{H}$ NMR $\left(500 \mathrm{MHz}, \mathrm{THF} \mathrm{d}_{8},-78{ }^{\circ} \mathrm{C}\right)-47.18\left(\mathrm{vt}, 1 \mathrm{H},{ }^{2} \mathrm{~J}_{\mathrm{PH}}=12 \mathrm{~Hz}, H-\mathrm{Ir}\right), 1.00-1.50$ (m, 36H, P-C $\left(\mathrm{CH}_{3}\right)_{3}$ ), 2.5-3.04 (m, 2H, P-CH $\mathrm{CH}_{2} \mathrm{Py}$ ), 3.70 (br t, 1H, P-CHPy), 5.67 (br d, 1H, Py$H$ ), 6.45-6.85 (m, 5H, Aryl-H, Py-H), 8.01 (br d, 2H, Aryl-H). ${ }^{13} \mathrm{C}\left\{{ }^{1} \mathrm{H}\right\}$ NMR $(500 \mathrm{MHz}$, THF $\mathrm{d}_{8},-78{ }^{\circ} \mathrm{C}$ ): 30.00 (m, P-C $\left.\left(\mathrm{CH}_{3}\right)_{3}\right), 39.0$ (br m, $\left.\mathrm{CH}_{2} \mathrm{P}\right), 65.30$ (br m, CH-P), 95 (br s, Py$\mathrm{CH}), 112$ (m, Aryl-CH), 122.4 (m, Aryl-CH), 123.3 (m, $\mathrm{C}_{\mathrm{ipso}}$ ), 127.6 (m, Aryl-CH), 131.3 (m, Py-CH), 144.7 (m, Aryl-CH),158.6 (s, Py-CC), 170.3 (m, Py-CC). The structure was confirmed by DEPT, C-H correlation experiment and 2D COSY NMR. Irradiating the hydride signal resulted in an NOE effect with the protons at $8.02 \mathrm{ppm}$.

\section{Reaction of $\left[\operatorname{Ir}(\mathrm{PNP})(\mathrm{D})\left(\mathrm{C}_{6} \mathrm{D}_{5}\right)\right]\left[\mathrm{PF}_{6}\right]$ (5a) with ${ }^{\mathrm{t}} \mathrm{BuOK}$ at $-78{ }^{\circ} \mathrm{C}$. Formation of $\operatorname{Ir}\left(P_{N P}^{*}\right)\left(C_{6} D_{6}\right)(D)(3 a)$ and $\operatorname{Ir}\left(d_{1}-P N P\right)\left(C_{6} D_{5}\right)(4 a)$.}

To a THF solution of $\left[\operatorname{Ir}(\mathrm{PNP})(\mathrm{D})\left(\mathrm{C}_{6} \mathrm{D}_{5}\right)\right]\left[\mathrm{PF}_{6}\right] 5 \mathbf{5 a}(15 \mathrm{mg}, 0.0185 \mathrm{mmol})$ at $-78{ }^{\circ} \mathrm{C}$ was added ${ }^{\mathrm{t}} \mathrm{BuOK}$ ( $2 \mathrm{mg}, 0.0185 \mathrm{mmol}$ ) resulting in an immediate color change to dark brown. The ${ }^{31} \mathrm{P}$ NMR spectrum revealed an $\mathrm{AB}$ quartet, implying the lack of a symmetry plane as a result of deprotonation of the benzylic protons. No hydride was seen in the ${ }^{1} \mathrm{H}$ NMR spectrum. ${ }^{2} \mathrm{H}$ NMR reveals a deuteride at $-47 \mathrm{ppm}$. Upon warming to room temperature complex 4a was observed. According to the integration in ${ }^{1} \mathrm{H}$ NMR, the deuteride was incorporated in the benzylic position.

\section{Reaction of $\left[\operatorname{Ir}(\mathrm{PNP})(\mathrm{H})(\mathrm{CO})\left(\mathrm{C}_{6} \mathrm{H}_{5}\right)\right]\left[\mathrm{PF}_{6}\right]$ with ${ }^{\mathrm{t}} \mathrm{BuOK}$. Formation of $\operatorname{Ir}\left(\mathrm{PNP}^{*}\right)\left(\mathrm{C}_{6} \mathrm{H}_{6}\right)(\mathrm{H})(\mathrm{CO})(6)$.}

To a THF solution of $\left[\operatorname{Ir}(\mathrm{PNP})(\mathrm{CO})(\mathrm{H})\left(\mathrm{C}_{6} \mathrm{H}_{5}\right)\right]\left[\mathrm{PF}_{6}\right]^{1}(15 \mathrm{mg}, 0.0178 \mathrm{mmol})$ at $25{ }^{\circ} \mathrm{C}$ was added ${ }^{t} \mathrm{BuOK}$ ( $2 \mathrm{mg}, 0.0178 \mathrm{mmol}$ ) resulting in an immediate color change to dark brown. The ${ }^{31} \mathrm{P}$ NMR spectrum revealed an $\mathrm{AB}$ quartet, imdicating lack of a symmetry plane, as a result of deprotonation of the benzylic protons. Complex $\mathbf{6}$ was also prepared by reaction of $\mathbf{3}$ 
with one equivalent of $\mathrm{CO}$ at $-78{ }^{\circ} \mathrm{C} .{ }^{1} \mathrm{H}$ NMR revealed a hydride at $-7.8 \mathrm{ppm}$, typical for a hydride trans to a $\mathrm{CO}$ ligand.

6: ${ }^{31} \mathrm{P}\left\{{ }^{1} \mathrm{H}\right\}$ NMR (250 MHz, $\left.\mathrm{CD}_{2} \mathrm{Cl}_{2}\right): 45.5\left(\mathrm{~d},{ }^{2} \mathrm{~J}_{\mathrm{PP}}=264.6 \mathrm{~Hz}, P-\mathrm{Ir}-\mathrm{P}\right), 36.6\left(\mathrm{~d},{ }^{2} \mathrm{~J}_{\mathrm{PP}}=264.6\right.$ Hz, P-Ir-P). ${ }^{1} \mathrm{H}$ NMR (250 MHz, $\left.\mathrm{CD}_{2} \mathrm{Cl}_{2}\right)-7.81\left(\mathrm{dd}, 1 \mathrm{H},{ }^{2} \mathrm{~J}_{\mathrm{PH}}=16.75 \mathrm{~Hz},{ }^{2} \mathrm{~J}_{\mathrm{PH}}=16.87 \mathrm{~Hz}, \mathrm{H}-\right.$ Ir), 1.14-1.40 (m, 36H, P-C( $\left.\left.\mathrm{CH}_{3}\right)_{3}\right), 3.1-3.2$ (m, 1H, P-CH $\left.\mathrm{CH}_{2} \mathrm{Py}\right), 3.47-3.59$ (m, 1H, P-CH $\left.{ }_{2} \mathrm{Py}\right)$, $3.65\left(\mathrm{vt},{ }^{2} \mathrm{~J}_{\mathrm{PH}}=4.32 \mathrm{~Hz}, \mathrm{P}-\mathrm{CHPy}\right), 5.62\left(\mathrm{~d}, 1 \mathrm{H},{ }^{3} \mathrm{~J}_{\mathrm{HH}}=6.50 \mathrm{~Hz}, \mathrm{Py}-H\right), 6.18\left(\mathrm{~d}, 1 \mathrm{H},{ }^{3} \mathrm{~J}_{\mathrm{HH}}=8.80\right.$ $\mathrm{Hz}$, Py-H), 6.47 (m, 1H, Py-H), 6.76 (m, 3H, Aryl- $H), 7.80$ (m, 2H, Aryl- $H) .{ }^{13} \mathrm{C}\left\{{ }^{1} \mathrm{H}\right\}$ NMR (500 MHz, $\left.\mathrm{CD}_{2} \mathrm{Cl}_{2}\right): 30.00$ (m, P-C $\left.\left(\mathrm{CH}_{3}\right)_{3}\right), 39.10$ (m, $\left.\mathrm{CH}_{2} \mathrm{P}\right), 65.33$ (m, CH-P), 97 (d, $\left.{ }^{2} \mathrm{~J}_{\mathrm{PC}}=15.52 \mathrm{~Hz}, \mathrm{Py}-\mathrm{CH}\right), 112$ (m, Aryl-CH), 122.4 (m, Aryl-CH), 124.3 (m, $\mathrm{C}_{\mathrm{ipso}}$ ), 127.2 (m, Aryl-CH), 132.3 (m, Py-CH), 146.7 (m, Aryl-CH), 158.3 (s, Py-CC), 170.3 (m, Py-CC), 184.2 (br t, Ir-CO). The structure was confirmed by DEPT, C-H correlation experiment and 2D COSY NMR.

IR $v_{\mathrm{co}}=1986 \mathrm{~cm}^{-1}$

\section{Reaction of $\operatorname{Ir}(\mathrm{PNP})\left(\mathrm{C}_{6} \mathrm{H}_{5}\right)(4)$ with CO. Formation of $\operatorname{Ir}\left(\mathrm{PNP}^{*}\right)\left(\mathrm{C}_{6} \mathrm{H}_{6}\right)(\mathrm{H})(\mathrm{CO})(6)$.}

To a benzene solution of $\operatorname{Ir}(\mathrm{PNP})\left(\mathrm{C}_{6} \mathrm{H}_{5}\right) 4(10 \mathrm{mg}, 0.015 \mathrm{mmol})$, placed in an NMR tube, was bubbled $\mathrm{CO}(0.34 \mathrm{ml}, 0.015 \mathrm{mmol})$ at $25{ }^{\circ} \mathrm{C}$. The color changed immediately to red-brown, resulting in complex 6.

\section{Reaction of $\operatorname{Ir}(\mathrm{PNP})\left(\mathrm{C}_{6} \mathrm{H}_{5}\right)(4)$ with $\mathrm{H}_{2}$. Formation of $\operatorname{Ir}(\mathrm{PNP})\left(\mathrm{C}_{6} \mathrm{H}_{6}\right)(\mathrm{H})(\mathrm{H})$ (7).}

To a benzene solution of $\operatorname{Ir}(\mathrm{PNP})\left(\mathrm{C}_{6} \mathrm{H}_{5}\right) 4(10 \mathrm{mg}, 0.015 \mathrm{mmol})$, placed in an NMR tube, was bubbled $\mathrm{H}_{2}(0.3 \mathrm{ml}, 0.015 \mathrm{mmol})$. The solution was shaken under $\mathrm{H}_{2}$ at $25^{\circ} \mathrm{C}$ for 30 min until the color changed to pale yellow. Evaporation of the solvent resulted in an almost colorless solid complex 7.

7: ${ }^{31} \mathrm{P}\left\{{ }^{1} \mathrm{H}\right\}$ NMR $\left(\mathrm{C}_{6} \mathrm{D}_{6}\right): 54.00$ (s). ${ }^{1} \mathrm{H}$ NMR (500 MHz, $\left.\mathrm{C}_{6} \mathrm{D}_{6}\right)-8.28$ (vt, $2 \mathrm{H},{ }^{2} \mathrm{~J}_{\mathrm{PH}}=12 \mathrm{~Hz}$, $H$-Ir- $H$ ), 1.29 (vt, 36H, $\left.{ }^{3} \mathrm{~J}_{\mathrm{PH}}=6.10 \mathrm{~Hz}, \mathrm{P}-\mathrm{C}\left(\mathrm{CH}_{3}\right)_{3}\right), 3.08$ (vt, 4H, ${ }^{2} \mathrm{~J}_{\mathrm{PH}}=3.5 \mathrm{~Hz}, \mathrm{P}-\mathrm{CH}_{2} \mathrm{Py}$ ), $6.40\left(\mathrm{~d}, 2 \mathrm{H},{ }^{3} \mathrm{~J}_{\mathrm{HH}}=7.5 \mathrm{~Hz}\right.$, Py-H), $6.76\left(\mathrm{t}, 1 \mathrm{H},{ }^{3} \mathrm{~J}_{\mathrm{HH}}=6.8 \mathrm{~Hz}\right.$, Aryl-H), $7.05\left(\mathrm{t}, 1 \mathrm{H},{ }^{3} \mathrm{~J}_{\mathrm{HH}}=\right.$ $7.5 \mathrm{~Hz}$, Py-H), 7.12 (t, 2H, ${ }^{3} \mathrm{~J}_{\mathrm{HH}}=7.5 \mathrm{~Hz}$, Aryl- $H$ ), $8.53\left(\mathrm{~d}, 2 \mathrm{H},{ }^{3} \mathrm{~J}_{\mathrm{HH}}=7.5 \mathrm{~Hz}\right.$, Aryl- $H$ ). ${ }^{13} \mathrm{C}\left\{{ }^{1} \mathrm{H}\right\}$ NMR (400 MHz, $\left.\mathrm{C}_{6} \mathrm{D}_{6}\right): 29.31\left(\mathrm{~m}, \mathrm{P}-\mathrm{C}\left(\mathrm{CH}_{3}\right)_{3}\right), 35.45\left(\mathrm{vt},{ }^{2} \mathrm{~J}_{\mathrm{PC}}=10.26 \mathrm{~Hz}, \mathrm{P}-\right.$ $\left.\mathrm{C}\left(\mathrm{CH}_{3}\right)_{3}\right), 42.33$ (m, $\left.\mathrm{CH}_{2} \mathrm{P}\right), 117.56-119.16$ (s, Py-CH, Aryl-CH), 125.79-126.93 (s, Py-CH, Aryl-CH), 130.64 (t, $\left.{ }^{2} \mathrm{~J}_{\mathrm{PC}}=8.75 \mathrm{~Hz}, \mathrm{C}_{\mathrm{ipso}}\right), 150.22\left(\mathrm{~d},{ }^{2} \mathrm{~J}_{\mathrm{PC}}=15.32 \mathrm{~Hz}, \mathrm{Py}-\mathrm{CH}\right) 162.81$ (s, PyCC). The structure was confirmed by DEPT, C-H correlation experiment and 2D COSY NMR. An NOE effect was seen between the hydrides at $-8.82 \mathrm{ppm}$ and the protons at 8.53 ppm. 
Reaction of $\operatorname{Ir}(P N P)\left(C_{6} H_{5}\right)(4)$ with $D_{2}$. Formation of $\operatorname{Ir}\left(d_{1}-P N P\right)\left(C_{6} H_{6}\right)(D)(H)(7 a)$.

To a benzene solution of $\operatorname{Ir}(\mathrm{PNP})\left(\mathrm{C}_{6} \mathrm{H}_{5}\right) 4(10 \mathrm{mg}, 0.015 \mathrm{mmol})$, placed in an NMR tube, was bubbled $\mathrm{D}_{2}(0.3 \mathrm{ml}, 0.015 \mathrm{mmol})$. The solution was shaken under $\mathrm{D}_{2}$ at $25{ }^{\circ} \mathrm{C}$ for $30 \mathrm{~min}$ until the color changed to pale yellow. Evaporation of the solvent resulted in an almost colorless solid complex 7a.

The ${ }^{1} \mathrm{H}$ and ${ }^{31} \mathrm{P}$ NMR spectra are identical to those of complex 7, except for the incorporation of one deuterium into the benzylic group. The protons at $3.08 \mathrm{ppm}$ were integrated to $3 \mathrm{H}$ with a $20 \mathrm{sec}$ delay between pulses. Also, a hydride was observed with an integration of one.

Reference:

1. Ben-Ari, E.; Cohen, R.; Gandelman, M.; Shimon, L. J. W.; Martin, J. M. L.; Milstein, D., Organometallics 2006, 25, 3190 